\title{
Pediatric Hand and Wrist Fractures
}

\author{
Janice C.Y. Liao, MBBS, MRCS ${ }^{a}$, Alphonsus K.S. Chong, MBBS, FAMS ${ }^{a, b, *}$
}

\section{KEYWORDS}

- Pediatric hand fractures • Physeal injuries • Seymour fractures • Phalangeal neck fractures

- Metacarpal fractures $\bullet$ Scaphoid fractures $\bullet$ Distal radius fractures

\section{KEY POINTS}

- Closed fractures with no clinical malrotation or scissoring generally have a favorable outcome with conservative management.

- The possibility of a Seymour fracture should be considered in pediatric fingertip injuries. A true lateral radiograph facilitates diagnosis and the condition should be treated as an open fracture.

- Conservative treatment of AI Qattan type II phalangeal neck fractures is associated with remodeling of sagittal malalignment and translation.

- Patients with clinical signs of carpal fractures and normal radiographs should be immobilized. Patients with persistent pain at review after 10 days to 14 days with a normal repeat radiograph should have an MRI done.

- Sporting injuries can occur through high-energy trauma or repeated stress. Chronic wrist pain in gymnast or finger pain in climbers should be assessed radiographically for stress related physeal injuries. Conservative treatment with splinting and complete rest from training a usually sufficient.

\section{EPIDEMIOLOGY}

Hand and wrist fractures have been consistently reported as among the most common fractures in children. ${ }^{1-3}$ They are 3 times more common in boys and peak in the teenage years (Fig. 1). ${ }^{4}$ Forearm fractures compromise the largest proportion of fractures. ${ }^{1}$ In the hand, the proximal phalanx and the fifth ray are the most commonly affected bone and digit, respectively (Fig. 2). ${ }^{4}$ Distal phalangeal fractures tend to occur in toddlers starting to explore the world with their hands, making them susceptible to fingertip crush injuries from slammed doors. With increasing age, the distribution of fractures moves proximally, with median ages of 12 years for proximal phalanx fractures and 15 years for metacarpal fractures. This distribution corresponds to the increasing body weight and participation in sporting activities during the teenage years.

\section{CONSIDERATIONS IN THE PEDIATRIC POPULATION}

Epidemiologic studies generally use 17 years or 18 years of age as the limit for pediatric fractures. The unique considerations in the management of pediatric hand fractures, however, apply to children with open physes with the potential to remodel. In addition, the ability of a child to participate and cooperate in the management may

Disclosure: The authors have nothing to disclose.

a Department of Hand and Reconstructive Microsurgery, University Orthopaedics Hand and Reconstructive Microsurgery Cluster, National University Health System, 1E, Kent Ridge Road, Singapore 119228;

b Department of Orthopaedic Surgery, Yong Loo Lin School of Medicine, National University of Singapore, Singapore

* Corresponding author. Department of Hand and Reconstructive Microsurgery, University Orthopaedics Hand and Reconstructive Microsurgery Cluster, National University Health System, 1E, Kent Ridge Road, NUHS Tower Block, Level 11, Singapore 119228.

E-mail address: doscksa@nus.edu.sg 


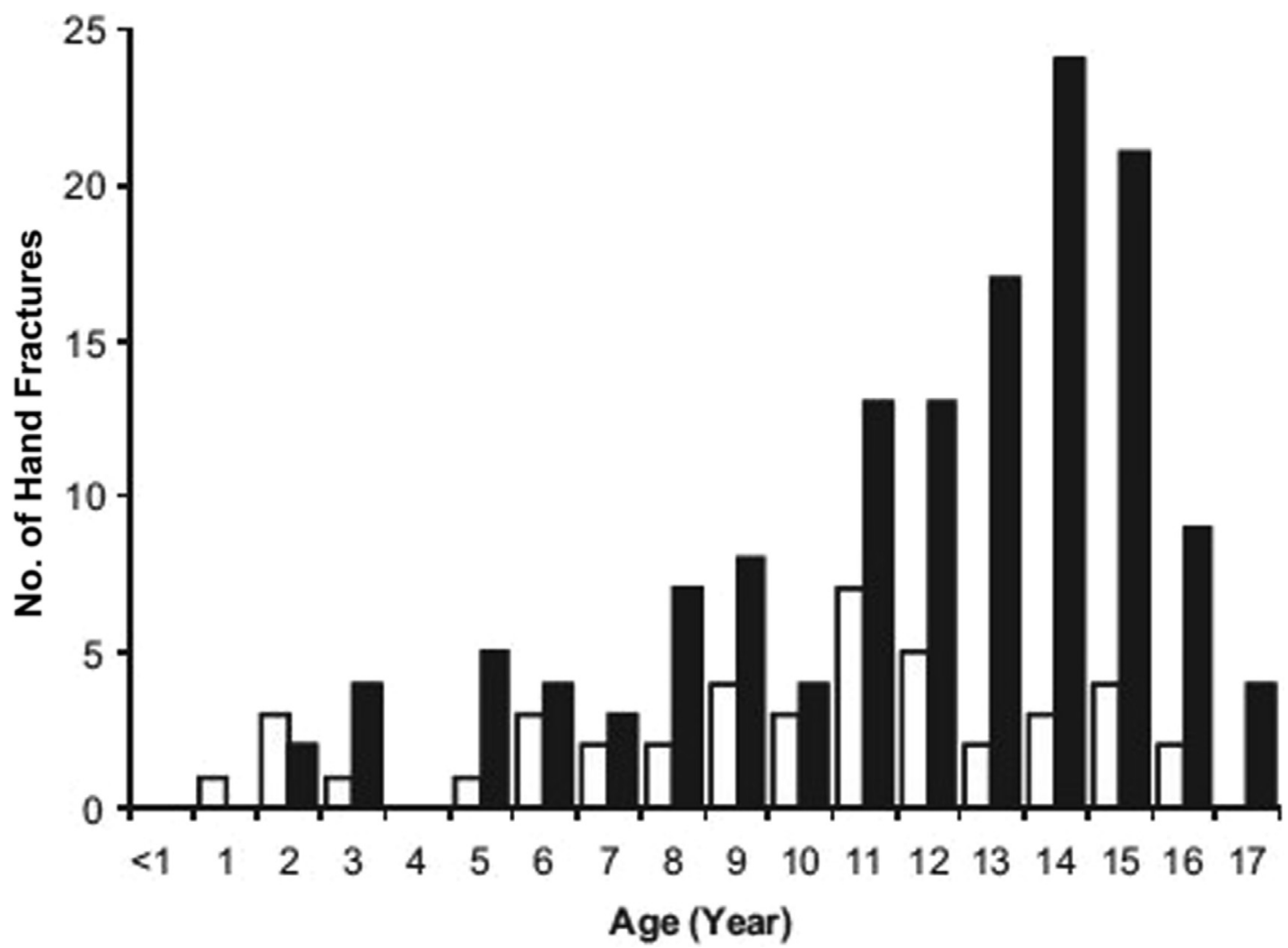

Fig. 1. The distribution of hand fractures by gender and age. White bars, girls; black bars, boys. (From Chew EM, Chong AK. Hand fractures in children: epidemiology and misdiagnosis in a tertiary referral hospital. J Hand Surg Am 2012;37(8):1686; with permission.)

affect the surgical indications and rehabilitation protocols.

\section{Physis}

In the pediatric population, fractures tend to occur through the physis because it is weaker than the surrounding bone and ligament. With a high healing potential, closed reduction needs to be done early. If delayed, percutaneous intrafocal pins may be required to aid reduction. ${ }^{5}$ Most pediatric hand fractures can be treated nonsurgically due to the remarkable potential of the physis to remodel. This remodeling occurs most reliably in the line of motion (flexion-extension), less in the coronal plane, and least in clinical malrotation and scissoring. Growth arrest occurs in 5\% to $10 \%$ of cases in patients with physeal fractures. ${ }^{6}$ It is rare after pediatric hand fractures, and length discrepancy has fewer functional implications compared with the larger long bones, especially in the lower limbs. ${ }^{7}$ Nevertheless, it is important to counsel patients and their parents of the potential sequelae of physeal injury, including shortening, angular deformity, and joint surface incongruity. ${ }^{8}$

\section{Surgical Considerations}

Open reduction of a dislocated physis should be performed after 1 or 2 failed attempts at closed reduction to minimize further injury to the physis. Intraoperatively, the physis should be handled atraumatically. Implants are not required for stable fractures postreduction. If a fracture remains unstable, the choice of implants should be physeal-friendly. Kirschner wires used should be the smallest size that provides adequate stability. Placement needs to be planned carefully to avoid multiple passes through the physis.

\section{Compliance with Treatment}

Cast immobilization is the treatment of choice in nonsurgical treatment of pediatric fractures due to fear of noncompliance. Compliance can vary greatly, and may not always correlate with the age of the child. Treatment should be 


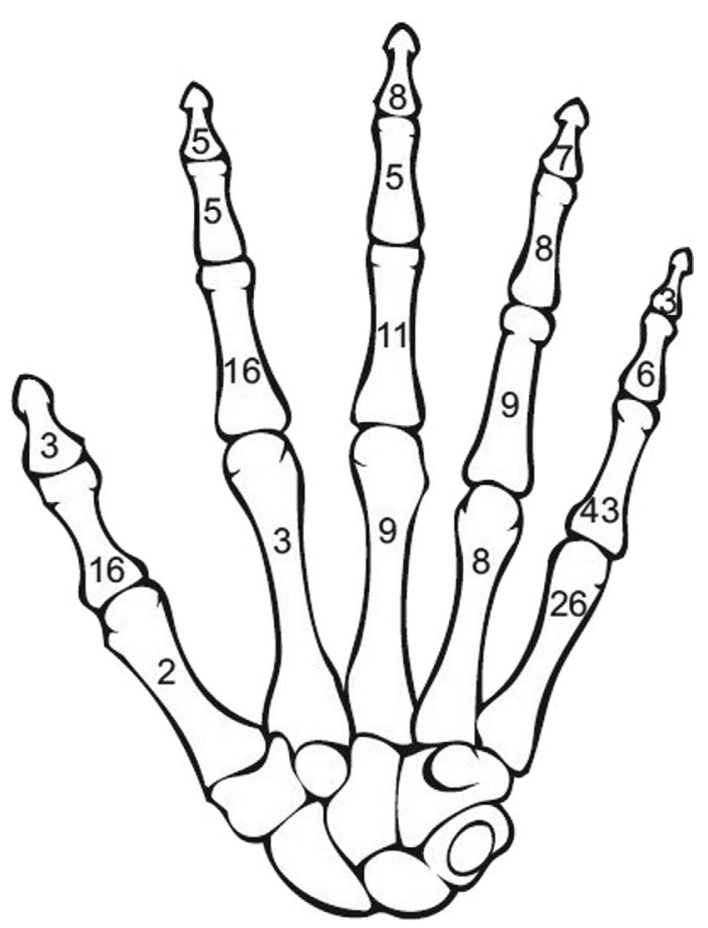

Fig. 2. The distribution of hand fractures by bone and ray. (From Chew EM, Chong AK. Hand fractures in children: epidemiology and misdiagnosis in a tertiary referral hospital. J Hand Surg Am 2012;37(8):1686; with permission.)

individualized and the ability of the child to cooperate can be gauged from discussion with the parents and involvement of the child in the management. In a sensible and cooperative child, splint immobilization can be considered to improve comfort and compliance with treatment. The authors have had good clinical results with splinting in appropriate patients.

\section{PHALANGEAL FRACTURES} Distal Phalanx

\section{Nail bed injuries and tuft fractures}

Tuft fractures usually result from a crush injury, most commonly from a door. ${ }^{9}$ These injuries often are associated with nail bed injuries and pulp lacerations (Fig. 3). When the nail is intact and adherent, the authors recommend nonoperative treatment regardless of hematoma size, presence of fracture, or injury mechanism. There is no significant difference in cosmetic outcome between operative treatment and conservative treatment. ${ }^{10}$ Injuries with disruption of the nail plate are treated with nail plate removal, débridement and irrigation, and nail bed repair. Nail replacement in the pediatric population has been shown to increase morbidity, including delayed wound healing and infection. ${ }^{11}$ The authors recommend nail replacement only in injuries involving the proximal nail fold to prevent the risk of synonychia and in fractures requiring an external splint. Nail bed repairs are commonly done with absorbable sutures (Fig. 4). Medical adhesives have been shown to achieve good outcome in terms of pain and appearance. $^{12}$ The use of medical adhesives significantly reduces the operative time, which is helpful in the pediatric population.

\section{Seymour fractures}

Seymour fractures are transepiphyseal SalterHarris fracture $(\mathrm{SH})$ type I or SH type II fractures of the distal phalanx with associated nail bed injury. It occurs in younger patients, with an average age of 8.7 years. ${ }^{13}$ The nail bed injury is often not apparent because the nail bed is avulsed proximally and interposed between the fracture site to preventing successful closed reduction. Clinically, patients may present with a
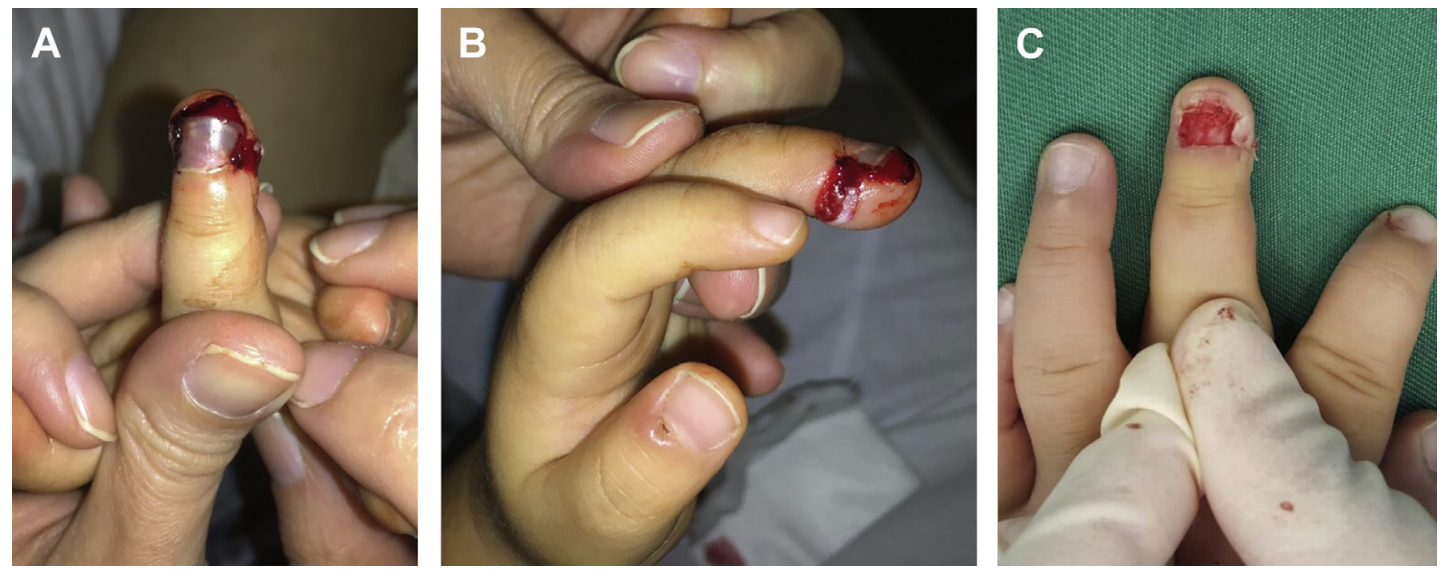

Fig. 3. $(A, B)$ Two-year-old patient with nail bed injury, pulp laceration, and tuft fracture sustained from a crush injury. (C) After nail plate removal and nail bed repair with Vicryl 6-0 sutures. 

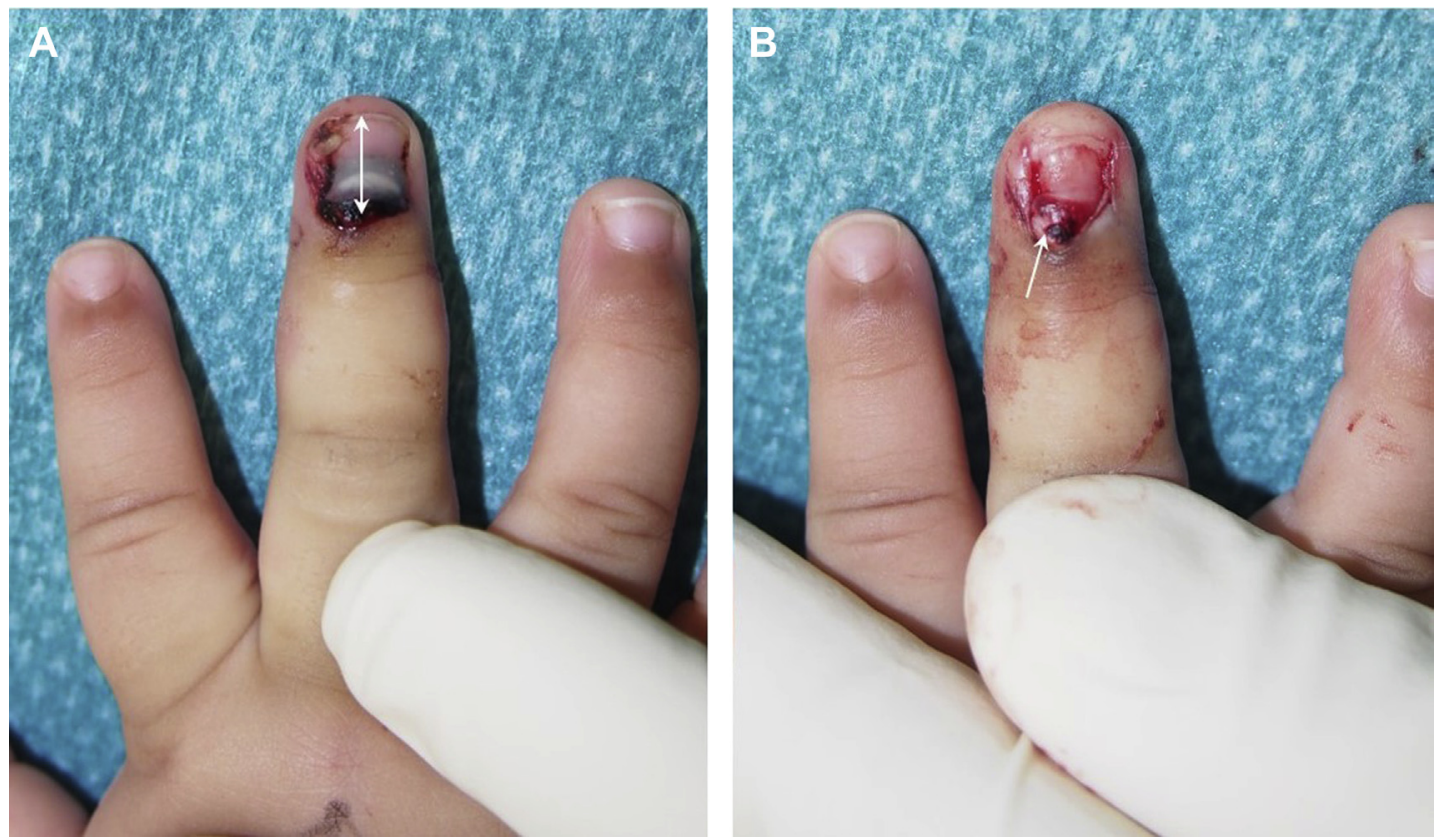

Fig. 4. (A) The appearance of a longer nail plate (white double arrow) suggests proximal nail bed avulsion. (B) After nail plate removal, nail bed was found to be interposed between the fracture site (white arrow).

pseudomallet deformity, with proximal nail plate sitting above the eponychium, which makes the nail plate appear longer (see Fig. 4). It is essential to be suspicious and always obtain true posteroanterior and lateral radiographs of the injured finger to confirm the diagnosis. Radiographs may show obvious displacement through the fracture or more subtle dorsal displacement of the epiphysis (Fig. 5). The authors use and recommend the principles of open fracture management (Box 1) (see Fig. 5). Missed diagnosis and delayed treatment are associated with increased risk of osteomyelitis and nail plate deformities. ${ }^{13}$

\section{Bony mallet}

There is paucity of literature on bony mallet in the pediatric population, and treatment is largely based on adults. In contrast to Seymour fractures, mallet fractures occur predominantly in patients older than 12 years of age. ${ }^{15}$ Axial injury with forced flexion of an extended distal interphalangeal joint (DIPJ) results in avulsion of terminal tendon, with a fragment of epiphysis recognized as an SH type III or SH type IV fracture. In adult mallet fractures, there are studies to support both conservative and surgical treatment. ${ }^{16}$ For nonoperative treatment, compliance with splint
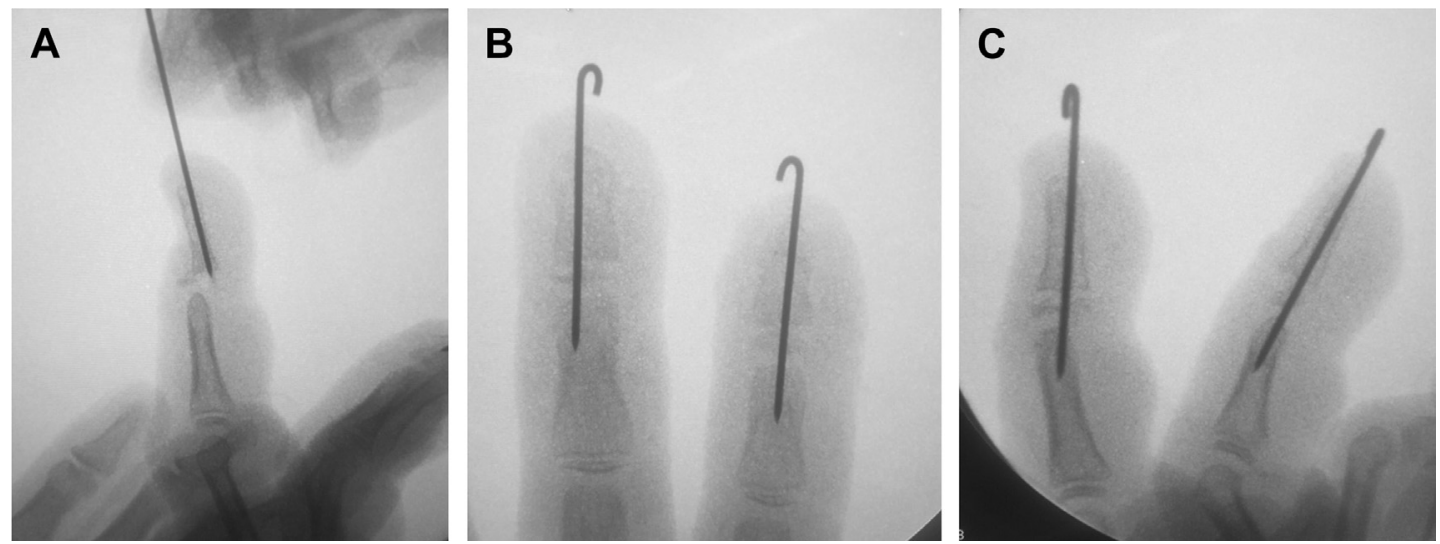

Fig. 5. (A) Lateral radiograph showing dorsal displacement of the epiphysis in a Seymour fracture. The Kirschner wire was used to aid the reduction. $(B, C)$ Fracture reduced and DIPJ transfixed. 
Box 1

Treatment protocol for Seymour fractures

- Intravenous antibiotics as soon as feasible

- Nail plate removal in operating theater

- Extrication of interposed nail bed

- Débridement and irrigation

- Reduction of fracture

- Assess stability-a majority can be treated with 4 weeks of splinting

- $20 \%$ of unstable fracture require DIPJ pinning with a single Kirschner wire ${ }^{14}$

maybe feasible because patients tend to be older. The splint can be reinforced with a boxing glove dressing, but the skin needs to be checked regularly. Surgical indications are listed in Box 2. Good clinical and radiological outcomes with minimal complications in 51 pediatric mallet fractures, both acute and chronic, have been reported. ${ }^{17}$

\section{Middle and Proximal Phalanx}

\section{Phalangeal neck fractures}

Phalangeal neck fractures are unique to the pediatric population, accounting for $13 \%$ of pediatric finger fractures. ${ }^{18}$ These fractures typically present with an apex volar angulation with dorsal displacement of the phalangeal head (Fig. 6). They are inherently unstable and believed to have poor remodeling potential due to the increased distance from the physis. ${ }^{19}$ These fractures are classified by Al Qattan into type I undisplaced, type II displaced with some bone-tobone contact, and type III displaced with no bone-to-bone contact. ${ }^{14}$ Troublesome phalangeal neck fractures are associated with poor prognostic factors and result in an unsatisfactory outcome despite surgical treatment (Box 3). ${ }^{20}$ Although surgical treatment of type II fractures has been common practice, ${ }^{5,14}$ several reports have shown remarkable remodeling of phalangeal neck fractures with nonoperative treatment. In 8 patients who were treated nonoperatively for phalangeal neck fracture malunion, fractures
Box 2
Surgical indications for bony mallet
- Fracture fragments involving greater than $50 \%$ of the articular surface
- Volar subluxation of the fracture fragment
- Issues with splint compliance

were remodeled with correction in the sagittal plane from $30^{\circ}$ to $0^{\circ}$ and coronal plane from $10^{\circ}$ to $5^{\circ} .{ }^{21}$ Sagittal translation also corrected from $58 \%$ at injury to $0 \%$ at final follow-up. All patients achieved functional range of motion and only 2 reported cosmetic deformities in the coronal plane. In the authors' institution, type II fractures without scissoring or obvious coronal deformity are treated nonoperatively. A recent review of these patients showed that there was significant improvement in the sagittal angulation and translation (see Fig. 6). It is imperative, however, to follow-up these patients with repeat radiographs. The authors treated a 4-year-old patient with a type II fracture, which converted to a type III fracture 2 weeks postinjury in a cast requiring closed reduction and percutaneous pinning (Fig. 7).

\section{Proximal phalangeal extraoctave fractures}

SH type II fractures of the proximal phalanx are the most common hand fractures. ${ }^{4}$ The fifth ray is most commonly injured from a hyperabduction injury, also known as an extraoctave fracture. This fracture typically result in an apex volar and radial deformity (Fig. 8). Due to the extraoctave fracture proximity to the physis, remarkable remodeling takes place and surgical treatment is rarely required. In the authors' center, approximately $25 \%$ of patients who presented to the Emergency Department with an extraoctave fracture underwent manipulation and reduction in the emergency department either by the emergency physicians or hand surgery residents. Only $2 \%$ of the patients with clinical malrotation and/or scissoring underwent surgery. Deformity should be assessed with fingers in midflexion because malrotation or scissoring easily can be missed with fingers in extension (Fig. 9). Close reduction and percutaneous pinning is the preferred surgical treatment. Although good cosmetic and functional recovery is expected at 1 year postsurgery, there is an initial $5 \%$ complication rate of infection, pin site complications, malunion, and stiffness. ${ }^{22}$

\section{METACARPAL FRACTURES}

Metacarpal fractures are common in children, with $80 \%$ involving the fifth metacarpal neck. ${ }^{18}$ The median age of children sustaining metacarpal fracture is 15 years, which is nearing skeletal maturity. ${ }^{4}$ Therefore, the management of these fractures is similar to that in the adult population. The key indications for surgical treatment are clinical malrotation and scissoring. Associated fight bite injuries also must be excluded. Various acceptable apex dorsal angulation has been reported. In general, the tolerance for angulation increases from index 

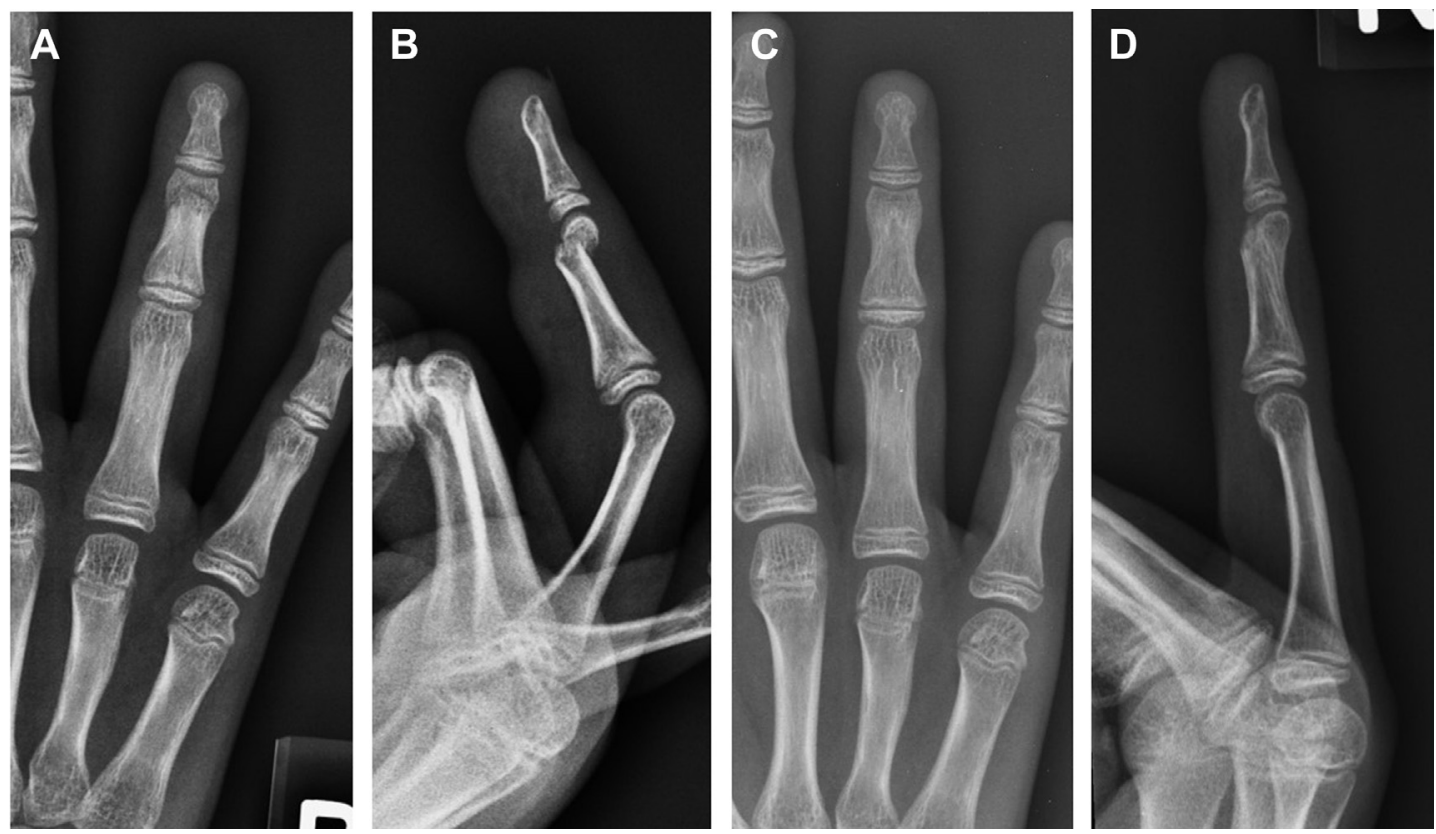

Fig. 6. Radiographs of a 12-year-old patient with right ring finger phalangeal neck fracture. $(A, B)$ Residual apex volar angulation and dorsal displacement post closed reduction. $(C, D)$ Significant remodeling at 3 months after injury.

finger to little finger and in younger patients with higher potential for remodeling. An acceptable degree of $10^{\circ}, 20^{\circ}, 30^{\circ}$, or $40^{\circ}$ angulation in the index through the small finger metacarpals is a reasonable guide. ${ }^{23}$ The authors have had good clinical results treating most pediatric metacarpal neck fractures nonoperatively. A recent randomized controlled trial showed that a hand-based thermoplastic splint improved early range of motion and grip strength with no significant difference in complications and noncompliance compared with the ulnar gutter plaster splint. ${ }^{24}$

\section{CARPAL FRACTURES}

Carpal fractures are uncommon in children but can be difficult to diagnose due to nonspecific clinical symptoms and incomplete ossification
Box 3
Poor prognostic factors for phalangeal neck fractures
- Vascular compromise
- Partial amputation
- Comminution of the phalangeal head
- Concomitant epiphyseal fracture
- All Al-Qattan Type III fractures

of the carpal bones. Similar to adults, scaphoid fractures are the most common followed by capitate fractures. ${ }^{25}$ Other carpal fractures are rare. Carpal fractures often result from high-energy trauma. In a review of pediatric all-terrain vehicles injuries, 32 hand fractures were composed of 15 metacarpal, 10 phalanx, 3 scaphoid, and 1 perilunate fracture dislocations and 1 pisiform, 1 hamate, and 1 triquetrum fractures. ${ }^{26}$ Another series looking at 61 children with suspected carpal fractures found 14 scaphoid fractures, 4 capitate fractures, 3 triquetral fractures, and 1 trapezoid bone bruising. ${ }^{27}$ The authors recommend immobilization of all patients with clinical signs of carpal fractures even with normal radiographs. Patients with persistent pain during review at 10 days to 14 days postinjury and a normal repeat radiograph should have an MRI done (Fig. 10). Nonoperative treatment with cast immobilization has a high-success union rate, with mean healing time of 56 days with no complications. ${ }^{27}$

\section{Acute Scaphoid Fractures}

Scaphoid fractures are the most common carpal fractures in children, with an increasing incidence in the past 2 decades. A recent epidemiology study quoted an incidence of 11 per 100,000 per annum. ${ }^{28}$ The average age of incidence in boys was 12.2 years and in girls was 10.3 years. 

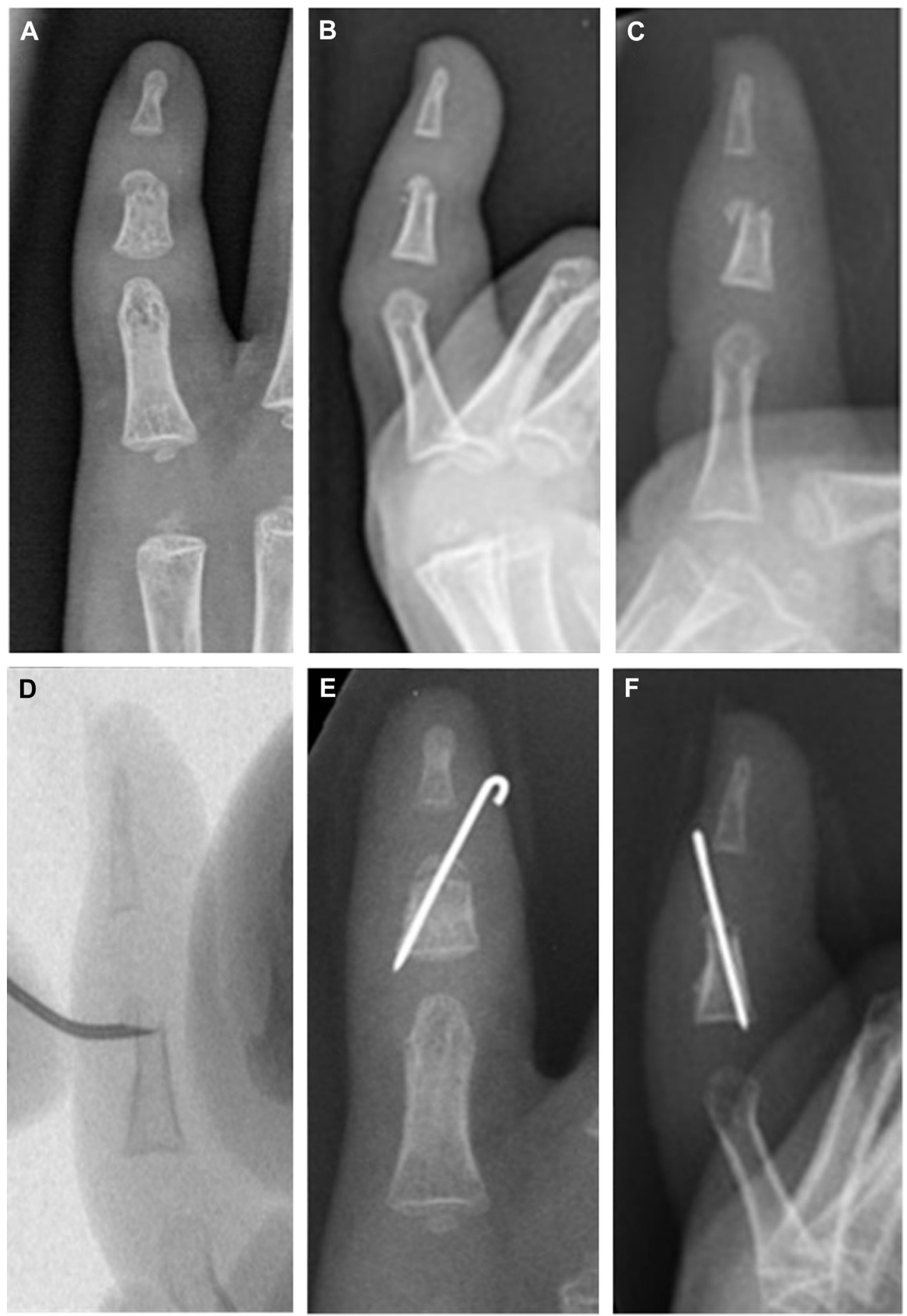

Fig. 7. $(A, B)$ The initial radiographs for a 4-year-old patient with left little finger phalangeal neck fracture. (C) At 2 weeks showing fracture displacement with significant dorsal angulation and epiphyseal dissociation with bayonet deformity. $(D, E, F)$ Close reduction was performed with the aid of intrafocal elevation using a 23-gauge needle and fixed with a 0.7-mm Kirschner wire. 


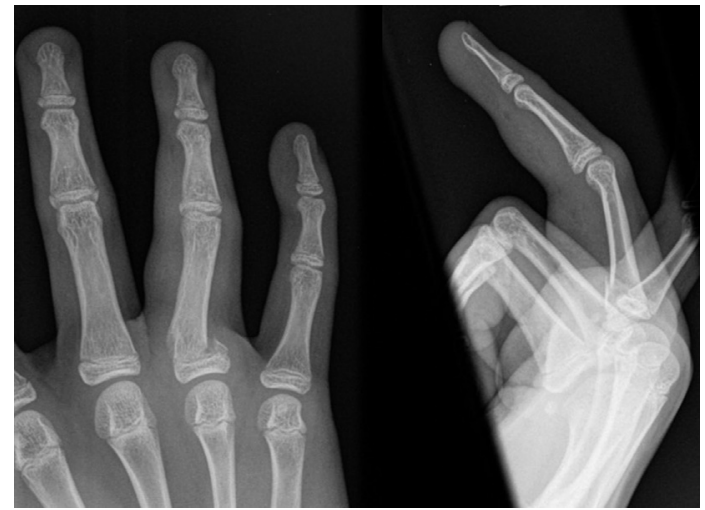

Fig. 8. Radiographs of a 12-year-old patient with right ring finger proximal phalanx base fracture with apex radial angulation (Left is PA view, Right is lateral view).

No scaphoid fractures were observed in boys below the age of 11 years and in girls below the age of 9 years. Historically, pediatric scaphoid fractures tend to occur at the distal pole and generally heal well. Recent analysis showed that increasing body mass index produces a fracture pattern similar to the adult population, with waist fractures the most common followed by distal pole fractures and proximal pole fractures. ${ }^{29}$ Cast immobilization of acute scaphoid fracture has been shown to achieve union rates of more than $90 \%$ in the pediatric and adolescent populations. ${ }^{29}$ The duration of immobilization depends on the fracture location, displacement, and chronicity. Waist fractures require longer immobilization with radiological healing between 5 weeks and 16 weeks compared with distal pole fractures, which take 4 weeks to 8 weeks. ${ }^{30}$ The authors recommend assessing patients at 4 weeks to 6 weeks for clinical and radiological healing and to continue with casting or splinting for another 4 weeks to 6 weeks if fracture has not united. If fracture union still cannot be confirmed at 12 weeks, a CT scan can be done to assess fracture healing.

\section{Chronic Scaphoid Fractures/Nonunion}

Natural history of scaphoid nonunion in the pediatric population is not well understood. Dorsal intercalated segment instability deformity does not necessarily occur after nonunion and may not be corrected with surgical fixation of the scaphoid. ${ }^{30}$ Pediatric scaphoid nonunion, however, has not been reported to progress to scaphoid nonunion advanced collapse. A 23\% union rate has been reported for chronic scaphoid fractures (defined as presentation $>6$ weeks from injury) treated with casting. The authors treated a 16-year-old patient who opted for conservative treatment of his scaphoid fracture, which eventually united after 3 years (Fig. 11). All chronic fractures or nonunions treated with surgical fixation with or without bone graft achieved a union rate of $95 \% .^{31}$

\section{Capitate Fractures}

Pediatric capitate fractures are not well studied. The capitate is the first carpal bone to ossify, rendering it more plastic and possibly more vulnerable to fracture compared with other carpal bones. A review of 53 patients with capitates fractures compared 43 adult patients to 10 pediatric patients under 16 years of age. ${ }^{25}$ The mean age of the pediatric population was 13 years. In children, isolated fractures were more common, whereas in adults, carpal fractures were more commonly associated with perilunate greater arc injury. Only 2 of 10 patients were treated surgically. One developed a nonunion requiring revision surgery. All pediatric patients achieved functional range of
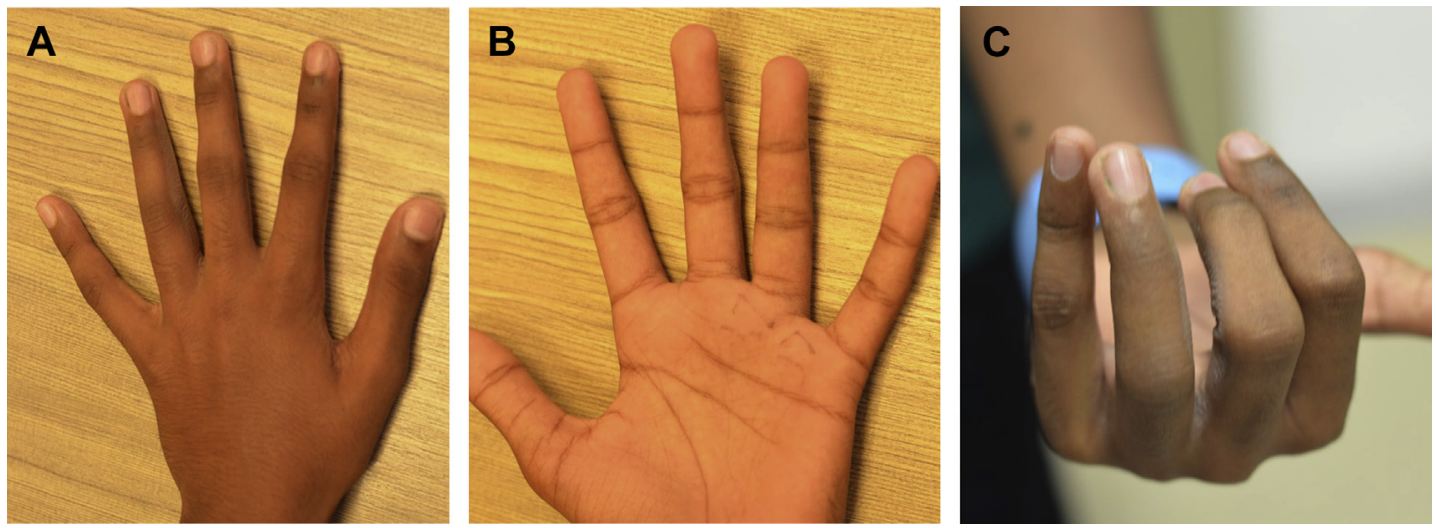

Fig. 9. $(A, B)$ Left ring finger appears normal in full extension. (C) Malrotation and scissoring are evident only in midflexion of the fingers (Left: dorsal view; Middle: palmar view; Right: midflexion view). 

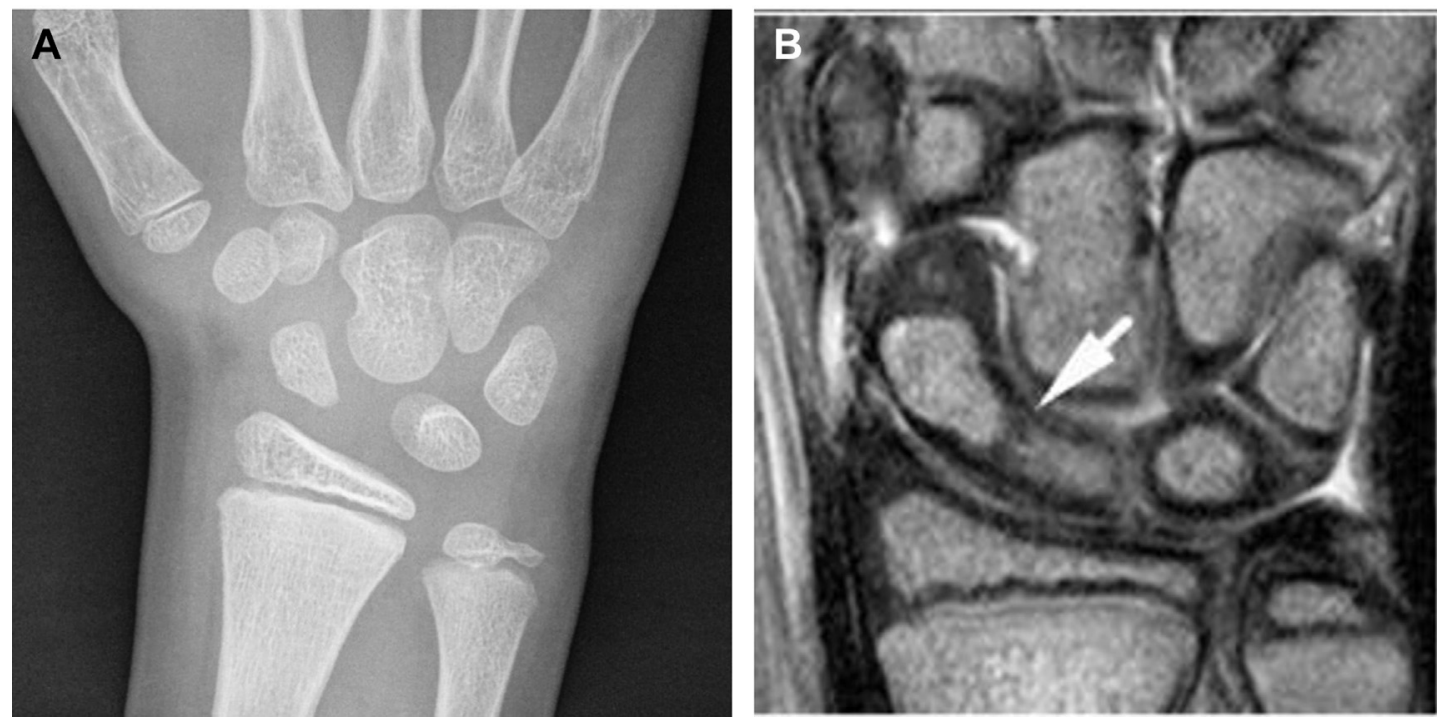

Fig. 10. (A) Radiograph of a 9-year-old boy with right thumb and wrist pain after a fall with tenderness in the anatomic snuffbox showed incomplete enchondral ossification of the scaphoid. $(B)$ MRI showed an undisplaced scaphoid waist fracture (arrow). Tip of arrow denotes the fracture site. (Courtesy of Dr Foo Tun Lin, Raffles Hospital, Singapore.)

motion at latest follow-up, in contrast to $48 \%$ in adults.

\section{DISTAL RADIUS FRACTURES}

Distal radius fractures are the most common pediatric fractures, comprising $20 \%$ of all pediatric fractures. ${ }^{32}$ They are more common in boys and peak in the teenage years. ${ }^{33} \mathrm{~A}$ majority of pediatric distal radius fractures are torus fractures resulting from a compression force with buckling of the concave side. Greenstick fractures occur from a bending force with unicortical break of the convex side. These fractures are inherently stable and can be treated with a short period of immobilization with a cast or splint and with no difference in outcome. ${ }^{34}$

Bicortical metaphyseal fractures often are displaced and are treated with closed reduction and cast immobilization if the alignment is unacceptable. Varying radiological parameters defining an acceptable alignment have been reported. ${ }^{35}$ In general, remodeling potential is higher closer to the physis, in the plane of motion and with more remaining years of growth. The authors prefer a short arm cast because it has been shown as effective as a long arm cast in maintaining reduction, with better ability to perform activities of daily living and fewer days of missed school. ${ }^{36}$ Although one-third of the distal radius fractures requiring close reduction resulted in late redisplacement, equivalent clinical outcome has been reported for distal radius fractures treated with closed reduction and cast immobilization versus initial pin fixation. ${ }^{37}$

The treatment principles of transphyseal fractures are similar to those of metaphyseal fractures, with additional considerations to minimize physeal trauma. Reduction maneuvers should be performed gently, with adequate analgesia and with multiple attempts avoided. The overall risk of physeal arrest has been reported to be $4 \%{ }^{36}$ Fractures with less than $15^{\circ}$ of angulation and up to $1 \mathrm{~cm}$ of shortening have been reported to fully remodel with no functional impairment. ${ }^{38}$

\section{SPORTING INJURIES}

Increased participation in sports and at a higher level in conjunction with heavier weight of the patients contribute to increasing sporting injuries in the teenage population. Pediatric hand and wrist fractures can occur acutely, through sports that result in high-energy trauma, or chronically, through repeated stress injury. The rates of acute hand/wrist injuries have been reported to be the highest in football, followed by similar rates in softball, wrestling, basketball, and hockey. Injuries occur from contact with another player, contact with a playing apparatus, or contact with the playing surface. ${ }^{38}$ All-terrain vehicles also have been reported to be associated with high incidence of upper extremity fractures, including rare carpal fractures, such as perilunate fracture dislocation and pisiform, hamate, and triquetral fractures. ${ }^{26}$ The recent emerging hoverboard has been 

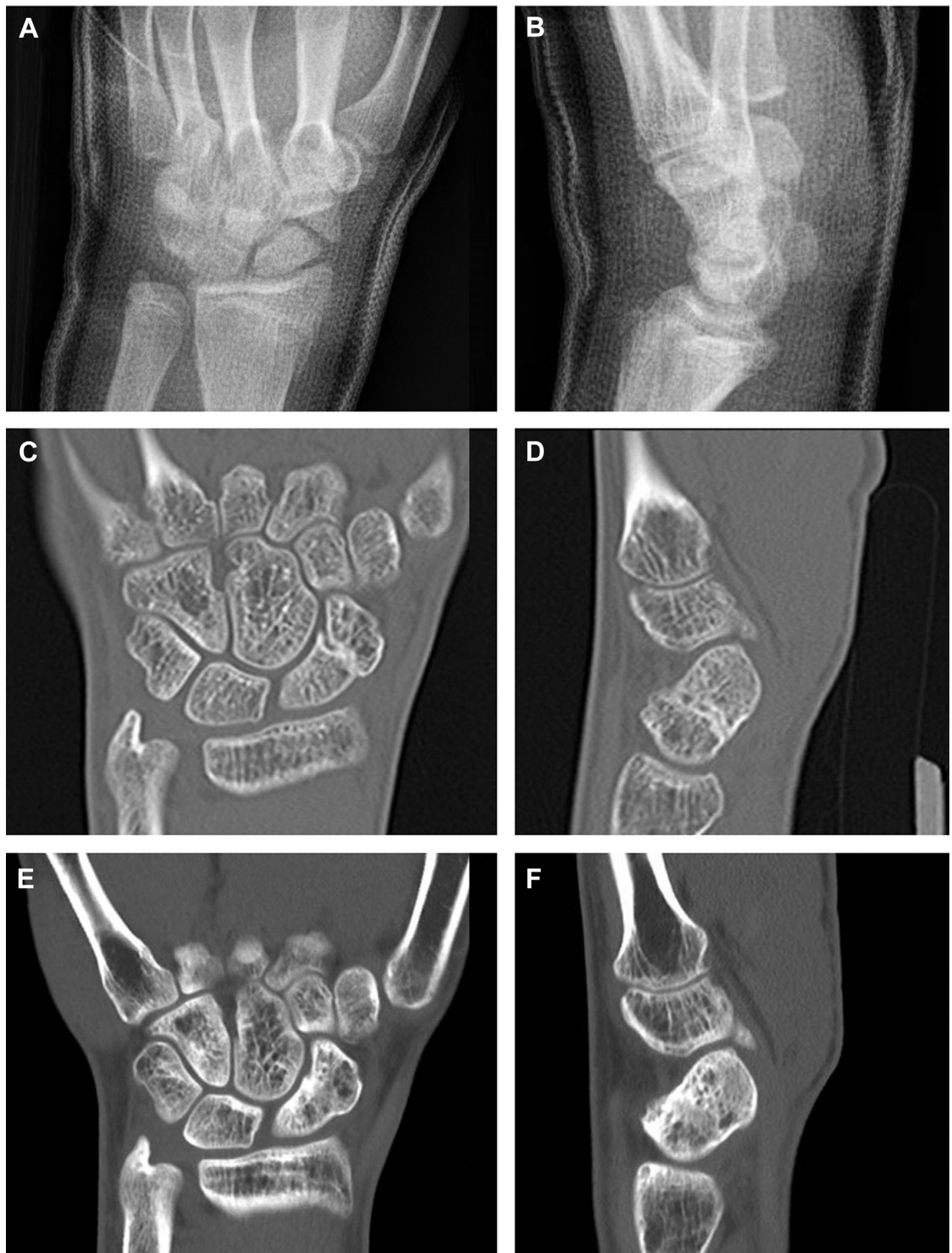

Fig. 11. $(A, B)$ Radiographs on presentation showing distal radius and scaphoid waist fractures. $(C, D) C T$ scan done at 1 year postinjury showing scaphoid waist nonunion. $(E, F)$ Repeat scan done at 3 years postinjury showing fracture union.

reported to cause more upper extremity fractures than other childhood recreational activities. ${ }^{39,40}$

Unlike acute fractures from sporting injuries, repeated stress injuries often are overlooked. Chronic wrist pain is commonly seen in gymnastics and other sports that involve repeated loading across the distal radius physis. Patients report dorsal wrist pain aggravated by 


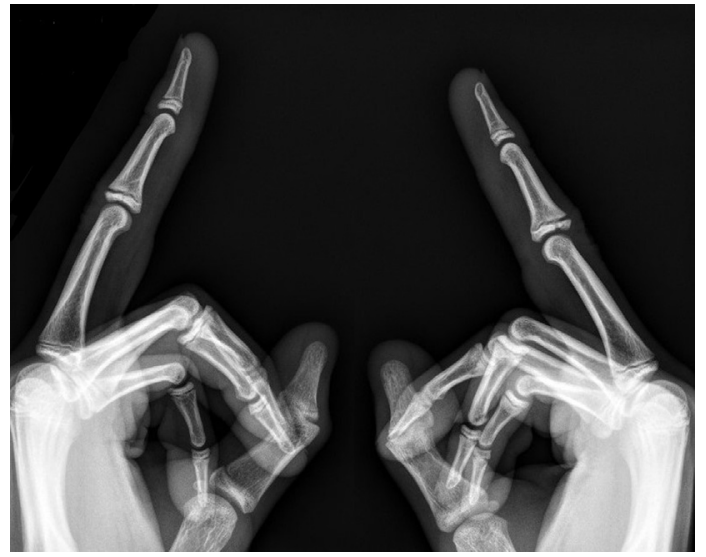

Fig. 12. A teenage rock climber with bilateral middle finger middle phalanx SH type III fractures that healed with rest and immobilization (left and right lateral views respectively).

loading the wrist joint. Radiograph shows stressrelated physeal injury, including widening and beaking of the physis, cystic changes of the metaphysis, and increased ulnar variance. ${ }^{41}$ Treatment involves 4 weeks to 6 weeks of resting, splinting, and training modification. Another notable repeated stress injury is middle phalanx base epiphyseal fracture in elite adolescent climbers. This is usually an SH type III fracture involving the middle finger or ring finger and more often associated with crimp grip (Fig. 12). ${ }^{42}$ In acute injuries, a majority of patients achieve excellent results with nonoperative treatment involving complete rest from climbing and gentle active range of motion until fracture healing. ${ }^{42}$ Recovery in delayed presentations with chronic nonunion is less predictable, with risks of osteonecrosis with resultant pain, permanent deformity and stiffness. ${ }^{43}$

\section{SUMMARY}

Pediatric hand and wrist fractures are common. Distal fractures like Seymour fractures and phalangeal neck fractures occur more commonly in younger patients and are unique to the pediatric population. Proximal fractures like metacarpal fractures tend to occur in teenage years, with increasing body weight and participation in sporting activities, and have more resemblance to adult fractures. Understanding of the physeal anatomy and carpal ossification is important in the accurate diagnosis of pediatric fractures. Consideration of the physeal biology and compliance with treatment are central to the management of these fractures. A majority have a favorable outcome with nonoperative treatment.
Operative treatment should be considered in patients with clinical malrotation and scissoring, open fractures, and significant fracture displacement. Physeal-friendly surgical approaches and implants should be used to minimize the sequelae of physeal injury.

\section{REFERENCES}

1. Chung KC, Spilson SV. The frequency and epidemiology of hand and forearm fractures in the United States. J Hand Surg Am 2001;26(5):908-15.

2. Hastings $\mathrm{H}$ 2nd, Simmons BP. Hand fractures in children. A statistical analysis. Clin Orthop Relat Res 1984;188:120-30.

3. Worlock PH, Stower MJ. The incidence and pattern of hand fractures in children. J Hand Surg $\mathrm{Br}$ 1986;11(2): 198-200.

4. Chew EM, Chong AK. Hand fractures in children: epidemiology and misdiagnosis in a tertiary referral hospital. J Hand Surg Am 2012;37(8):1684-8.

5. Matzon JL, Cornwall R. A stepwise algorithm for surgical treatment of type II displaced pediatric phalangeal neck fractures. J Hand Surg Am 2014;39(3): 467-73.

6. Dabash S, Prabhakar G, Potter E, et al. Management of growth arrest: current practice and future directions. J Clin Orthop Trauma 2018;9(Suppl 1): S58-66.

7. Culp RW, Osgood JC. Posttraumatic physeal bar formation in the digit of a child: a case report. J Hand Surg Am 1993;18(2):322-4.

8. Shapiro F. Epiphyseal growth plate fractureseparations: a pathophysiologic approach. Orthopedics 1982;5(6):720-36.

9. Satku M, Puhaindran ME, Chong AK. Characteristics of fingertip injuries in children in Singapore. Hand Surg 2015;20(3):410-4.

10. Roser SE, Gellman H. Comparison of nail bed repair versus nail trephination for subungual hematomas in children. J Hand Surg Am 1999;24(6):1166-70.

11. Miranda BH, Vokshi I, Milroy CJ. Pediatric nailbed repair study: nail replacement increases morbidity. Plast Reconstr Surg 2012;129(2):394e-6e.

12. Edwards S, Parkinson L. Is fixing pediatric nail bed injuries with medical adhesives as effective as suturing?: a review of the literature. Pediatr Emerg Care 2019;35(1):75-7.

13. Reyes BA, Ho CA. The high risk of infection with delayed treatment of open seymour fractures: salter-harris I/II or juxta-epiphyseal fractures of the distal phalanx with associated nailbed laceration. J Pediatr Orthop 2017;37(4):247-53.

14. Al-Qattan MM. Extra-articular transverse fractures of the base of the distal phalanx (Seymour's fracture) in children and adults. J Hand Surg Br 2001;26(3): 201-6. 
15. Lankachandra M, Wells CR, Cheng CJ, et al. Complications of distal phalanx fractures in children. J Hand Surg Am 2017;42(7):574.e1-e6.

16. Wehbe MA, Schneider LH. Mallet fractures. J Bone Joint Surg Am 1984;66(5):658-69.

17. Reddy M, Ho CA. Comparison of percutaneous reduction and pin fixation in acute and chronic pediatric mallet fractures. J Pediatr Orthop 2019;39(3): 146-52.

18. Mahabir RC, Kazemi AR, Cannon WG, et al. Pediatric hand fractures: a review. Pediatr Emerg Care 2001;17(3):153-6.

19. Green DP. Hand injuries in children. Pediatr Clin North Am 1977;24(4):903-18.

20. Al-Qattan MM, Al-Munif DS, AlHammad AK, et al. The outcome of management of "troublesome" vs "non-troublesome" phalangeal neck fractures in children less than 2 years of age. J Plast Surg Hand Surg 2016;50(2):93-101.

21. Puckett BN, Gaston RG, Peljovich AE, et al. Remodeling potential of phalangeal distal condylar malunions in children. J Hand Surg Am 2012;37(1): 34-41.

22. Boyer JS, London DA, Stepan JG, et al. Pediatric proximal phalanx fractures: outcomes and complications after the surgical treatment of displaced fractures. J Pediatr Orthop 2015;35(3):219-23.

23. Cornwall R. Finger metacarpal fractures and dislocations in children. Hand Clin 2006;22(1):1-10.

24. Davison PG, Boudreau N, Burrows R, et al. Forearmbased ulnar gutter versus hand-based thermoplastic splint for pediatric metacarpal neck fractures: a blinded, randomized trial. Plast Reconstr Surg 2016;137(3):908-16.

25. Kadar A, Morsy M, Sur YJ, et al. Capitate fractures: a review of 53 patients. J Hand Surg Am 2016;41(10): e359-66.

26. Shannon SF, Hernandez NM, Sems SA, et al. Pediatric orthopaedic trauma and associated injuries of snowmobile, ATV, and dirtbike accidents: a 19-year experience at a level 1 pediatric trauma center. J Pediatr Orthop 2018;38(8):403-9.

27. Eckert K, Trobs RB, Schweiger B, et al. Diagnostically approach to pediatric carpal fractures: a retrospective analysis. Z Orthop Unfall 2016;154(1):43-9 [in German].

28. Ahmed I, Ashton F, Tay WK, et al. The pediatric fracture of the scaphoid in patients aged 13 years and under: an epidemiological study. J Pediatr Orthop 2014;34(2):150-4.

29. Gholson JJ, Bae DS, Zurakowski D, et al. Scaphoid fractures in children and adolescents: contemporary injury patterns and factors influencing time to union. J Bone Joint Surg Am 2011;93(13):1210-9.

30. Nellans KW, Chung KC. Pediatric hand fractures. Hand Clin 2013;29(4):569-78.

31. Jauregui JJ, Seger EW, Hesham K, et al. Operative management for pediatric and adolescent scaphoid nonunions: a meta-analysis. J Pediatr Orthop 2019; 39(2):e130-3.

32. Cheng JC, Shen WY. Limb fracture pattern in different pediatric age groups: a study of 3,350 children. J Orthop Trauma 1993;7(1):15-22.

33. Shah NS, Buzas D, Zinberg EM. Epidemiologic dynamics contributing to pediatric wrist fractures in the United States. Hand (N Y) 2015;10(2):266-71.

34. Boutis K, Willan A, Babyn P, et al. Cast versus splint in children with minimally angulated fractures of the distal radius: a randomized controlled trial. CMAJ 2010;182(14):1507-12.

35. Pretell Mazzini J, Rodriguez Martin J. Paediatric forearm and distal radius fractures: risk factors and re-displacement-role of casting indices. Int Orthop 2010;34(3):407-12.

36. Bae DS. Pediatric distal radius and forearm fractures. J Hand Surg Am 2008;33(10):1911-23.

37. Miller BS, Taylor B, Widmann RF, et al. Cast immobilization versus percutaneous pin fixation of displaced distal radius fractures in children: a prospective, randomized study. J Pediatr Orthop 2005;25(4):490-4.

38. Do TT, Strub WM, Foad SL, et al. Reduction versus remodeling in pediatric distal forearm fractures: a preliminary cost analysis. J Pediatr Orthop B 2003; 12(2):109-15.

39. Donnally CJ 3rd, Lawrie CM, Rush AJ 3rd, et al. The season of hoverboards: a case series of fractures. Pediatr Emerg Care 2017;33(5):325-8.

40. Kattan AE, AlShomer F, Alhujayri AK, et al. A case series of pediatric seymour fractures related to hoverboards: increasing trend with changing lifestyle. Int J Surg Case Rep 2017;38:57-60.

41. Mariscalco MW, Saluan P. Upper extremity injuries in the adolescent athlete. Sports Med Arthrosc Rev 2011;19(1):17-26.

42. Hochholzer T, Schoffl VR. Epiphyseal fractures of the finger middle joints in young sport climbers. Wilderness Environ Med 2005;16(3):139-42.

43. El-Sheikh Y, Lutter C, Schoeffl I, et al. Surgical management of proximal interphalangeal joint repetitive stress epiphyseal fracture nonunion in elite sport climbers. J Hand Surg Am 2018;43(6):572.e1-5. 